\title{
Autonomous House Floor Cleaning Robot
}

\author{
Chandan Kumar, Ashutosh Kumar Singh, Rajneesh Pandey
}

\begin{abstract}
The biggest household issue is the cleaning of house or surrounding are without wasting much time but the cleaning process is laborious and time taking process, but for maintaining hygiene in house, house must be cleaned regularly. So, for keeping in mind this issue in this paper an autonomous cleaning robot is discussed, this robot works on predefined algorithm for cleaning the floor and follow a particular path for cleaning the floor. The robot comprises a frame on which all the essential units of robot are mounted. The Bluetooth module for communicating with user, motors for providing movement, ultrasonic and VL53LOX for sensing surrounding environment, a water pump for providing vet cleaning with a cloth which is connected with a bar and rotated by a servo motor.
\end{abstract}

Keywords: robot, autonomous, manual control, communication, sensors, motors, water pump.

\section{INTRODUCTION}

The technology is improving with very fast pace in current period of time, currently autonomous system are developing in exponential rate[1]. The autonomous systems mostly comprises of Robots, which are electromechanical devices used in industrial and domestic application[2]. In this research paper a domestic cleaning robot is discussed for cleaning surrounding or desired are by controlling the device manually and autonomously. The robot is connected with the user device via Bluetooth module and haveArduino Uno as controller unit which controls all the functions of robot like vacuum cleaning, vet floor cleaning, sensing obstacles by using installed sensors for performing and completing give task.

\section{TECHNOLOGIES USED}

Arduino Uno: Arduino Uno is electronic device which can be programmed to control specific electrical units. The Arduino Uno have Atmega628P IC which can be programmed to control unit. The Arduino Uno can take input from various sensors and act accordingly. The Arduino Uno have digitalwrite pin for input and output in digital signal form and Analogwrite pin for input and output in analogue signal form[3]. The Arduino Uno is programmed by a software know as Arduino IDE by using $\mathrm{C}, \mathrm{C}++$ as programming language and uses pre-installed libraries for different components[4].

Revised Version Manuscript Received on 10 September, 2019.

Dr. Chandan Kumar, Department of Mechanical Engineering, Noida Institute of Engineering and Technology, Noida, U.P. India. (email: researchnietip@gmail.com)

Ashutosh Kumar Singh, Department of Electronics \& Communication Engineering, Noida Institute of Engineering and Technology, Noida, U.P. India. (email: researchnietip@gmail.com)

Rajneesh Pandey, Department of Mathematics, Noida Institute of Engineering and Technology, Noida, U.P. India. (email: researchnietip@gmail.com)

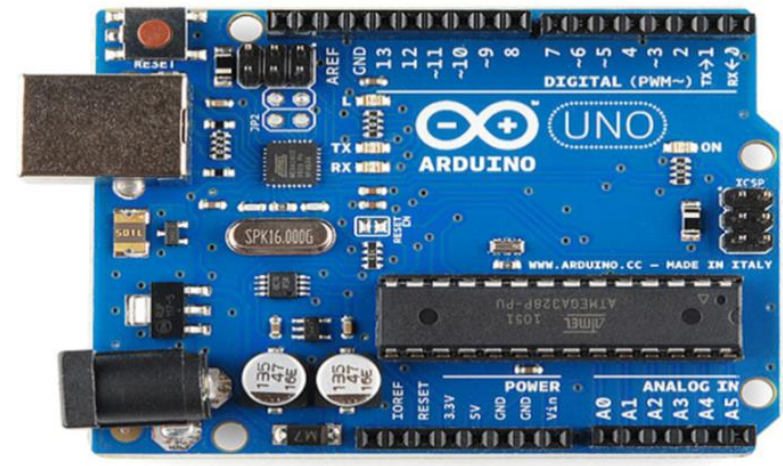

Figure 1: Arduino Uno

Ultrasonic Sensor: Ultrasonic sensor is used for measuring the distance between obstacle and the robot. The works on simple principle of sound propagation, the sensor have two units first is transmitter which transmits high frequency soundwaves and second unit is receiver which receives ultrasonic soundwaves which bounce back after hitting an object. The sensor is used in this Project is HC-SR04 which is connected with the ArduinoUno[5][6].

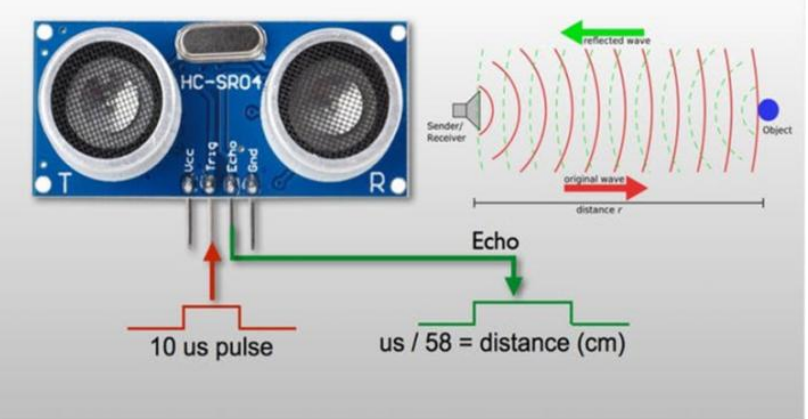

Figure 2: HC-SR04 Ultrasonic senor and its working

DC Geared Motor: The Geared DC motor is a combination of gear and DC motor. The geared assembly is used for increasing torque of motor and reducing RPM of motor. The geared are specifically designed for optimally using the output of geared motor[7].

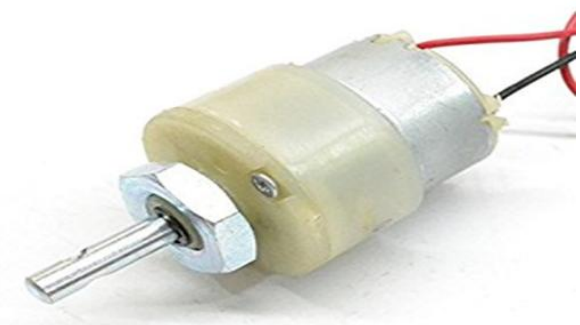

Figure 3: DC geared motor

Published By: Blue Eyes Intelligence Engineering \& Sciences Publication 


\section{Autonomous House Floor Cleaning Robot}

Vacuum Motor: The vacuum motor is a device which is combination of certain unit for sucking the air. The vacuum motor comprises a motor, a vacuum pump and a shaft. The motor is connected with pump via using the shaft. When the motor rotates, the pump creates a negative pressure on side and high pressure on other side by rotating and thus sucks the air inside and other small particles. The power of vacuum motor unit depends upon the perfect ratio of motor and vacuum pump[8][9].
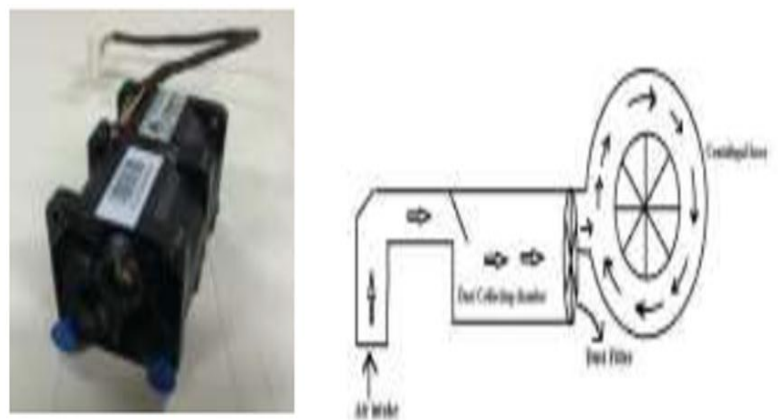

Figure 4: Vacuum Motor and its working

L298N Dual H-Bridge motor driver: The motor driver is electronic IC which is used for controlling the operations of motor like starting and stopping function of motor, for detecting the faults in motor, and other parameters which can affect the condition of motor[10].

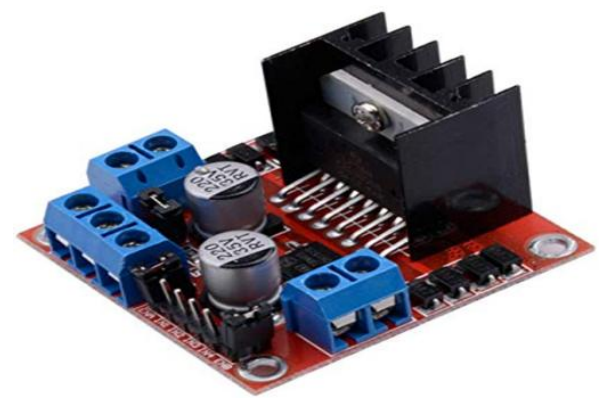

Figure 5: L298N H-Bridge Motor Driver

VL53L0X: The VL53L0X is sensor which detects time of flight distance. The sensor comprises a small leaser and a receiver which sensors the time of light to come back to sensor, The sensors also calculates the time of light travel. The sensor is implemented in this project for detecting the obstacles. It can detect objects between range $30 \mathrm{~mm}$ to $1000 \mathrm{~mm}[11]$.

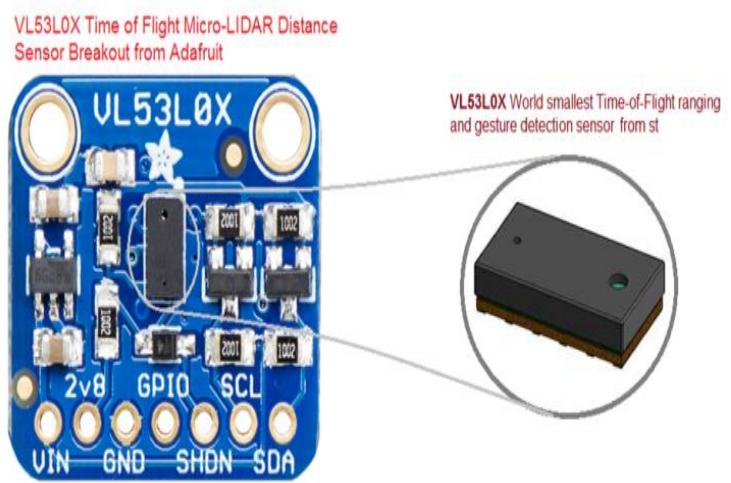

Figure 6: VL53L0X sensor Bluetooth HC-06
The HC-06 is a communication module which is used for small area communication. The HC-06 have ability to send and receive data. This module is connected [12] with robot for send and receiving data from user. The Bluetooth module give connectivity to device by using their MAC address.

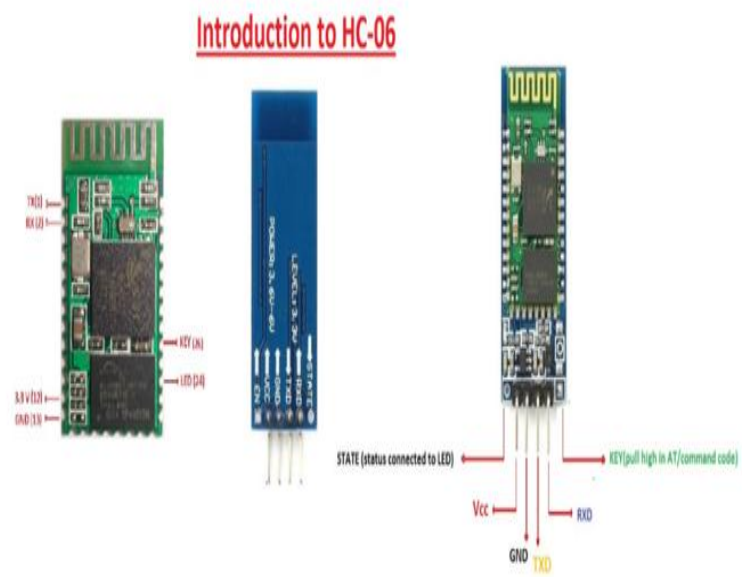

Figure 7: HC-06 Module

Submersible Pump: The submersible pump is associated with the rotor for vet cleaning the floor. The submersible pump is connected with a water containing unit, the pump operates on 5V DC power for smooth functioning[13].

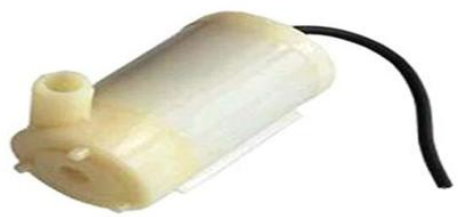

Figure 8: 5V submersible pump

\section{WORKING OF ROBOT}

The robot have two working principle first is Manual Mode and second is Autonomous mode.

In manual mode the robot is controlled by using a mobile application which is connected with robot by wireless communication using HC-06 Bluetooth module. The user can controlled the robot just like a remote control car using mobile phone. The mobile application provides directional control to robot for performing its operations.

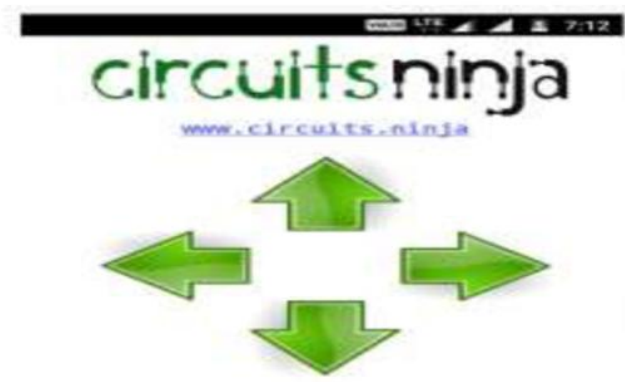

Figure 9: Android application User interface for manual control 
The second mode is autonomous in this mode robot uses a predefined algorithm and pre-defined path for cleaning the area. The robot uses the feedback from installed sensors which will direct the robot for performing its task.

The robot uses ultrasonic and VL53LOX sensor for determining the obstacle, whenever robot detects the obstacle it turn 90 left and again 90 right for continue following path.

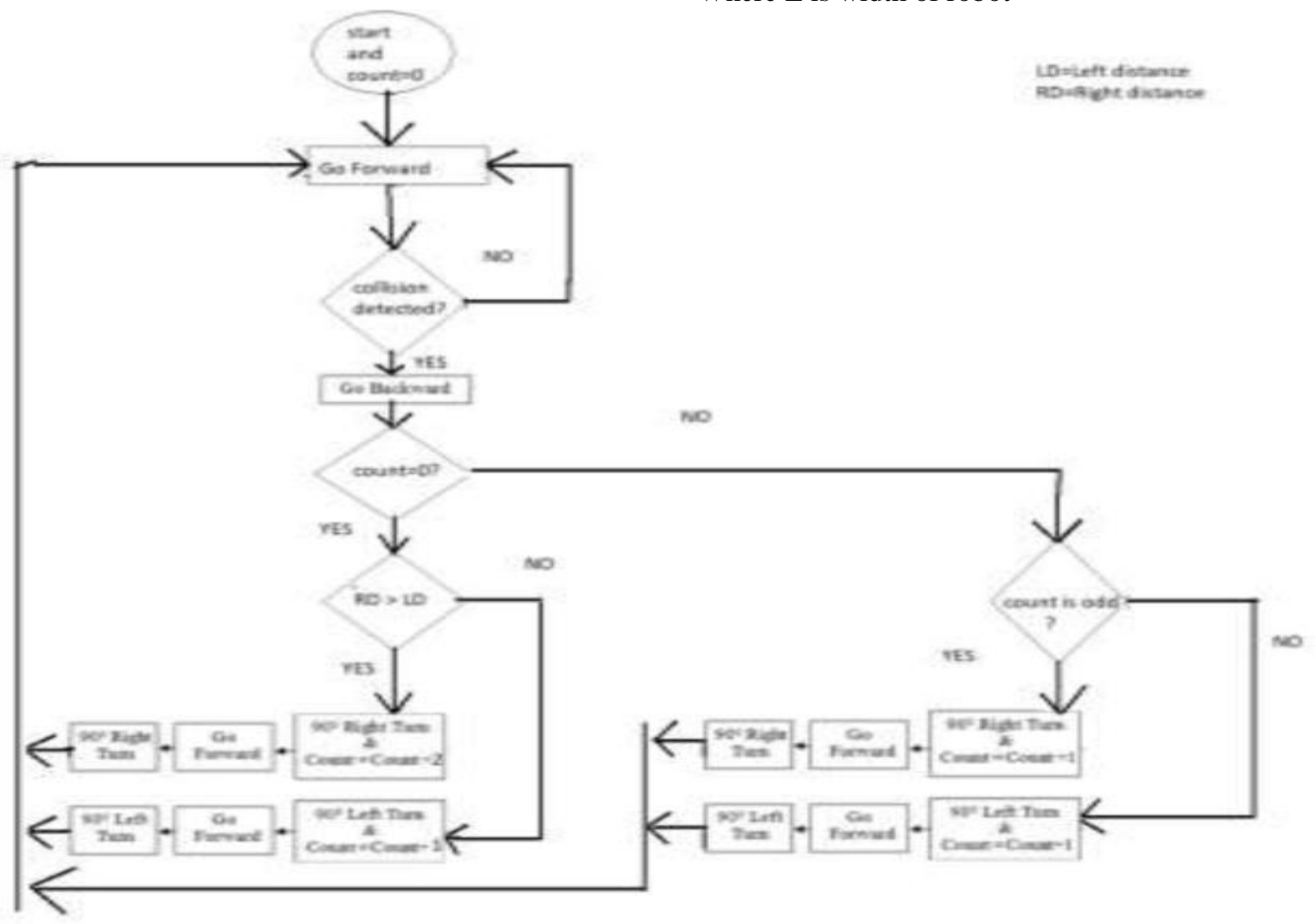

Figure 10: Path representation of robot

Where $\mathrm{L}$ is width of robot

Figure 11: Algorithm for autonomous robot control

\section{WORKING OF ROBOT}

When user starts the robot the microcontroller senses the environment around the robot and start working the first step robot perform is vacuum clean the surrounding area and after completing the vacuum clean the robot starts vet cleaning by using vet cleaning cloth connected on robot front side with a motor connected with Arduino Uno for performing vet cleaning operation. The robot uses "S" shaped path for cleaning and can be controlled manually to mobile phone via Bluetooth module.

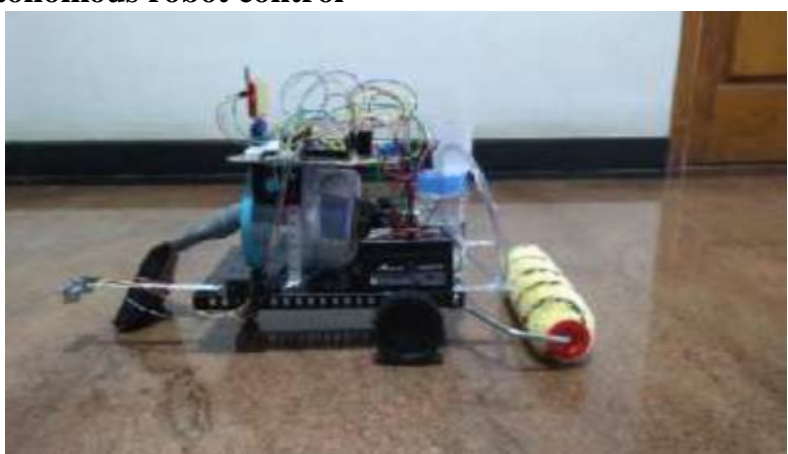

Figure 12: Prototype of Cleaning Robot 


\section{Autonomous House Floor Cleaning Robot}

\section{RESULT}

The robot is tested in two environments first is manual control in which the device is controlled by using mobile application by controlling the device reached the efficiency of $96.25 \%$.

The second mode is autonomous mode in this mode device is tested and worked autonomously according to algorithm by sensing surrounding area, the device is achieved an accuracy of $81.5 \%$.

The accuracy is determined by area cleaned by robot out of total area in 100 attempts.

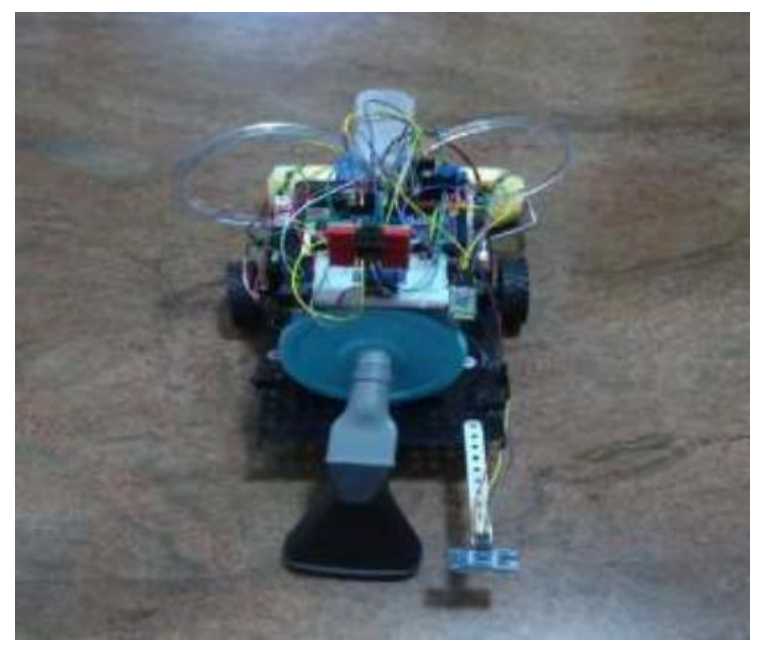

Figure 13: Autonomous Cleaning Robot top view

\section{CONCLUSION}

The device is worked as designed and planned, the user easily controlled the device and cleaned the surrounding are efficiently, as the device performed best in manual mode as compare to autonomous mode. In future the device will be embedded with a camera and high GPU and processing power unit for performing image processing and device will be trained by using machine learning and artificial intelligence technology for improving accuracy of device.

\section{REFERENCES}

1. R. Matthaei et al., "Autonomous driving," in Handbook of Driver Assistance Systems: Basic Information, Components and Systems for Active Safety and Comfort, 2015.

2. M. W. Spong, S. Hutchinson, and M. Vidyasagar, "Robot modeling and control," IEEE Control Systems. 2006.

3. D. R. Tobergte and S. Curtis, "Arduino Uno," J. Chem. Inf. Model., 2013.

4. D. Wheat and D. Wheat, "Arduino Software," in Arduino Internals, 2012.

5. P. Pirkonen and B. Ekberg, "Ultrasonic," in Progress in Filtration and Separation, 2014.

6. C. Scott, "HC-SR04 Ultrasonic Sensor," Arduino Basics, 2012. .

7. T. Verstraten, R. Furnemont, G. Mathijssen, B. Vanderborght, and D. Lefeber, "Energy Consumption of Geared DC Motors in Dynamic Applications: Comparing Modeling Approaches," IEEE Robot. Autom. Lett., 2016.

8. N. S. Harris, "VACUUM PUMP," in A-to-Z Guide to Thermodynamics, Heat and Mass Transfer, and Fluids Engineering, 2011.

9. S. Nakada, H. Yoshikawa, H. Ohno, T. Nishijima, and H. Kawakami, "Vacuum cleaner," J. Acoust. Soc. Am.,
2005.

10. AgusFaudin, "Tutorial Arduinomengakses driver motor L298N," AgusFaudin, 2017.

11. N. Lakovic, M. Brkic, B. Batinic, J. Bajic, V. Rajs, and N. Kulundzic, "Application of low-cost VL53LOX ToF sensor for robot environment detection," 2019.

12. Sayem, "AT Command Mode of HC-05 and HC-06 Bluetooth Module," Autodesk, 2016. .

13. G. Takacs, Electrical Submersible Pumps Manual. 2009. 